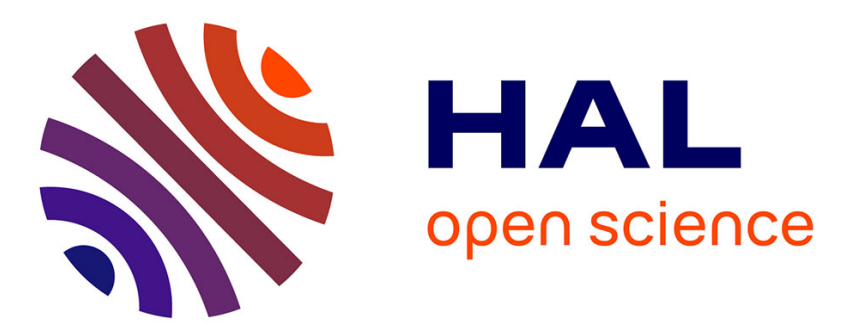

\title{
Analyse technologique de la méthode de débitage de l'abri Suard (La Chaise-de-Vouthon, Charente)
}

Anne Delagnes

\section{To cite this version:}

Anne Delagnes. Analyse technologique de la méthode de débitage de l'abri Suard (La Chaisede-Vouthon, Charente). PALEO : Revue d'Archéologie Préhistorique, 1990, 2, pp.81-88. halshs00447536

\section{HAL Id: halshs-00447536 \\ https://shs.hal.science/halshs-00447536}

Submitted on 15 Jan 2010

HAL is a multi-disciplinary open access archive for the deposit and dissemination of scientific research documents, whether they are published or not. The documents may come from teaching and research institutions in France or abroad, or from public or private research centers.
L'archive ouverte pluridisciplinaire $\mathbf{H A L}$, est destinée au dépôt et à la diffusion de documents scientifiques de niveau recherche, publiés ou non, émanant des établissements d'enseignement et de recherche français ou étrangers, des laboratoires publics ou privés. 


\title{
ANALYSE TECHNOLOGIQUE DE LA MÉTHODE DE DÉBITAGE DE L'ABRI SUARD (LA CHAISE-DF-VOUTHON, CHARENTE).
}

\author{
par Anne DELAGNES*
}

\begin{abstract}
Résumé : La méthode de débitage de l'abri Suard consiste en la production de séries successives d'enlèvements uni iolaires, réalisées soit à partir d'un même plan de frappe soit à partir de plans de frappe opposés ou orthogonaux. Sa place dans la variabilité des méthođics de débitage Levallois est discutée.

Abstract : The flaking method of the Abri Suard lies in the production of series of unidirectionnal removals ; these series are realized either from the same striking platform or from opposite or perpendicular striking platforms. Its position in the variability of the Levallois flaking methods is discussed.
\end{abstract}

L'analyse technologique d'un ensemble lithique permet d'aboutir non seulement à la détermination de faits et gestes techniques mais aussi à la reconstitution de leur enchainement tout au long d'une séquence de production. En l'absence de remontages cette vision dynamique des comportements techniques repose sur la lecture diacritique des nucléus et des produits de débitage, permettant d'établir l'agencement et l'ordre des enlèvements ainsi que leur rôle dans la séquence de débitage.

C'est ce type de démarche qui a guidé l'analyse de l'industric lithique de l'abri Suard présentée ici. Cette anlyse concerne le matériel issu des fouilles menées par André Debenath de 1967 et 1983 ets'insère dans une étude de l'ensemble de la chaîne opératoire.

Les analyses sédimentologiques et paléontologiques concordent pour situer le remplissage de l'abri Suard à la fin du Riss (Debenath, 1965 et 1974 ; David et Prat, 1965). Une série de datations absolues par Uranium-Thorium a permis de préciser ces données et de corréler l'ensemble de la séquence au stade isotopique 6 (Schwarcz et Debenath, 1979 ; Blackwell, Schwarcz et Debenath, 1983).

Cet assemblage lithique se rattache aux industries du Palćolithique moyen ancien et semble appartenir sur le plan typologique au Moustérien typique riche en racloirs (Debenath et Delagnes, à paraître).

Seuls les résultats de l'analyse de la couche 51 sont présentés dans cet article. Cette couche, située au milieu du remplissage de l'abri (constitué des couches 48 et 53), est la plus importante quantitativement et peut être considérée comme représentative de tout remplissage : les autres niveaux n'ont pas révélé de différence notable d'un point de vue technique.

En se fondant sur les travaux réalisés récemment par Eric Boëda sur le concept Levallois (Boëda, 1986 a et 1988b), il apparaît que la conception du débitage de l'abri Suard regroupe l'ensemble des critères de détermination du concept Levallois, qui sont les suivants :

1. exploitation d'une surface de débitage opposée à une surface d'aménagement des plans de frappe, ces deux surfaces étant délimitées par un plan d'intersection ;

2. mise en place de critères techniques de prédétermination (plans de frappe, convexités latérales et distales sur la surface de débitage);

3. débitage d'enlèvements parallèles au plan d'intersection des deux surfaces ;

4. emploi d'une méthode de production s'intégrant dans la variabilité des méthodes Levallois (récurrentes ou linéales) ;

5. technique de percussion directe au percuteur dur.

Bien que le débitage de l'abri Suard concorde tout à fait avec cette définition, il présente un certain nombre de particularités techniques qu'il conviendra de décrire avant de s'interroger sur sa position au sein du concept Levallois.

La reconstitution de la séquence opératoire a été facilitée par le fait que l'ensemble de la production est présent et que les nucléus ont été abandonnés à des stades d'exhaustion très variés.

Ces derniers $(\mathrm{N}=85)$ constituent près de $10 \%$ de la totalité des pièces. A l'issue de l'analyse technologique il s'avère que la méthode récurrente unipolaire est attestée sur $64 \%$ des nucléus (= 54 pic̀ces). Le reste des nucléus est constitué de catégories se situant chronologiquement en amont ou en aval des phases de plein débitage, c'est-àdire pour l'essentiel de nucléus abandonnés àl'état d'ébauches $(16 \%)$ et de nucléus indifférenciés (14\%). La méthode récurrente unipolaire est donc la méthode qui a régi l'ensemble de la production lithique. 


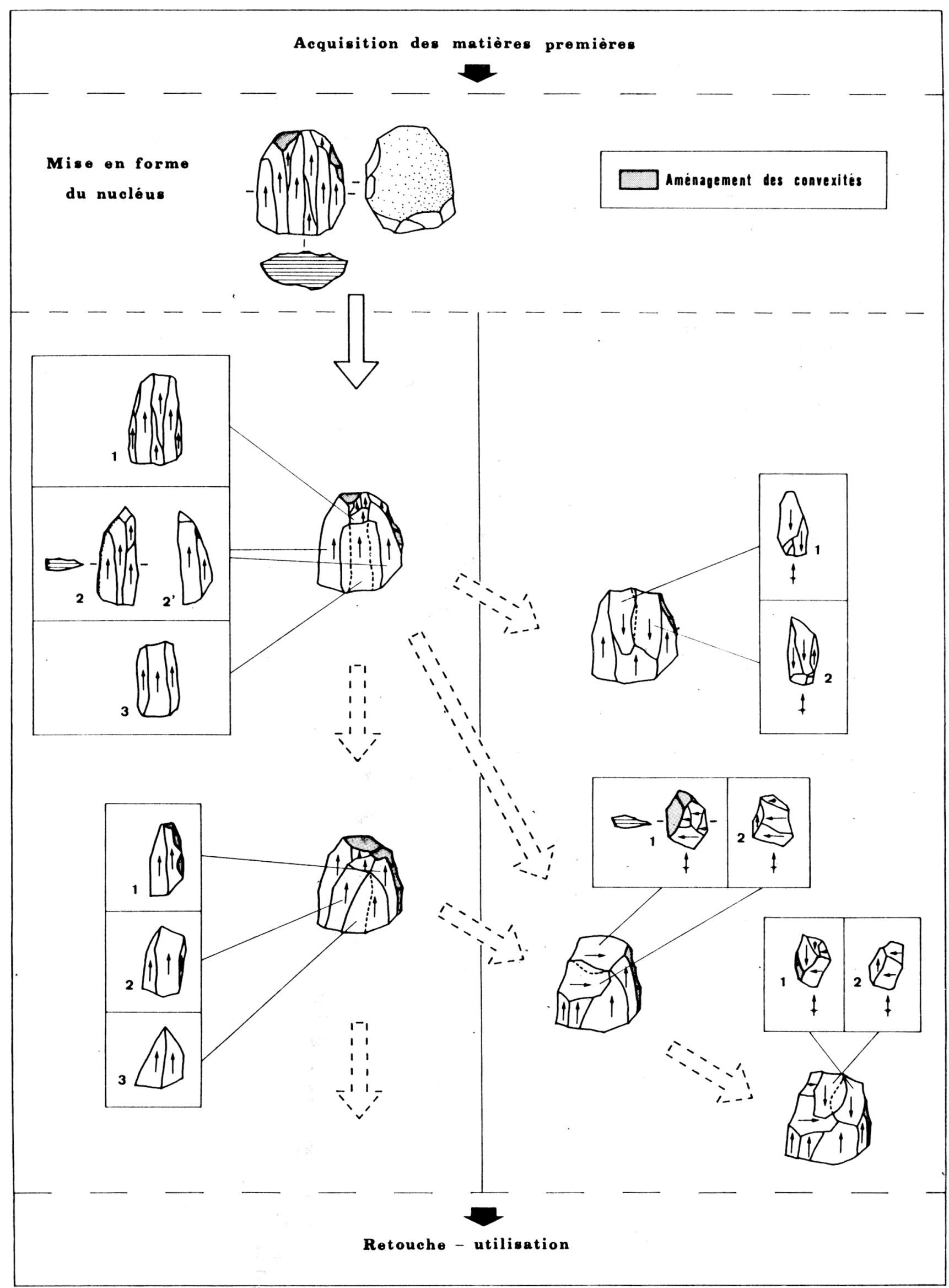

Fig. 1. Représentation schématique de la séquence de débitage de l'abri Suard (couche 51), La-Chaise-de-Vouthon. 


\section{DESCRIPTION DE LA SEQUENCE DEBITAGE} (fig. 1).

Tous les stades opératoires sont marqués par un comportement semblent procéder d' une conception économique du débitage.

- Dès le choix des supports à débiter, les tailleurs ont opté pour des supports dans la forme était déjà très proche de celle de nucléus préformés : il s'agit d'éclats importés ou produits sur place (supports reconnaissables pour $39 \%$ des nucléus récurrents unipolaires, ce taux étant à considérer comme une valeur minimale), et de blocs diaclasés peu épais. Ces derniers supports, lorsqu'ils ont été abandonnés à l'état d'ébauches, présentent des plans de diaclases qui, après l'aménagement d'un plan de frappe sommaire, ont été exploités de façon à ce que les premiers enlèvements filent le long des arêtes dégagées par ces pans.

- La mise en forme faisant suite à cette phase de sélection des supports semble avoir été assez succinte ; après la mise en place d'un plan de frappe, les tailleurs ont réalisé sur la face la plus plane du support une série d'enlèvements unipolaires très rapprochés les uns des autres; cette mise en forme semble s'être achevée lorsque cela s'avérait nécessaire, par quelques petits enlèvements en zone latéro-distale, régularisant la surface de débitage, créant ou accentuant des convexités. Un tel schéma de mise en forme correspond aux nucléus Levallois à préparation parallèle décrits par F. Bordes (1980).

La conséquence principale de ce type de préparation réside dans le fait que tous les éclats prédéterminés produits par la suite, et notamment ceux directement issus des phases de mise en forme (les éclats premiers), présentent des négatifs d'enlèvements antérieurs très majoritairement unipolaires.

- Les phases de plein débitage se caractérisent par la réalisation de séries successives d'enlèvements aux dépens d'une même surface de débitage. La récurrence consiste donc dans ce cas, non sculement en un débitage de plusieurs éclats prédéterminés successifs (constituant une séric), mais aussi en la production de plusieurs séries d'éclats. Au scin d'une même série les enlèvements sont strictement parallèles entre eux.

* La première série suivant le stade de mise en forme, est dans tous les cas une série unipolaire, réaliséc à partir du plan de frappe initial (probablement réaménagé entre temps). Les enlèvements produits sont au nombre de trois en moyenne.

A l'issue de cette première série, deux options possibles sont attestées par les nucléus :

* l'option qui semble avoir été dominante consiste en la réalisation d'une seconde série unipolaire de même sens, à partir du même plan de frappe, précédée d'un réaménagement minimal de la surface de débitage (par petits enlèvements réservés plutôt aux parties latérales des nucléus). Le nombre de séries pouvant avoir été produites successivement sclon ce même schéma est difficile à estimer.

* La seconde option est caractérisée par un changement d'orientation dans le débitage et par l'absence do réaménagement des convexités sur la surface de débitage d'une séric à l'autre. Le tailleur a fait pivoter son nucléus de 90 ou $180^{\circ}$ pour produire une autre séric sur la même surface de débitage après l'aménagement d'un nouveau plan de frappe. Il s'agit bien de séries distinctes produites successivement: chaque série comprend en moyenne deux à trois enlèvements unipolaires et se distingue aisément du fait qu'elle est rigoureusement parallèle (de sens opposé) ou orthogonale à la précédente. Ces séries sont le plus souvent au nombre de deux; dans quelques cas jusqu'à trois séries orthogonales sont visibles sur le nucléus. Cette option entraîne l'économie d'une remise en forme de la surface de débitage par l'utilisation, soit des convexités conservées en partie distale du nucléus à l'issue d'une séric unipolaire, soit des enlèvements de la série précédente ayant eux-mêmes dégagé de nouvelles convexités.

Si ce procédé semble avoir été rentable à court terme, par le passage direct d'une série à la suivante, il ne semble pas avoir permis une exploitation très durable des nucléus : au stade où été abandonnés les nucléus, les enlèvements des deuxièmes séries sont fréquemment rebroussés, ceux des troisièmes séries le sont encore plus souvent.

Le changement d'orientation dans le débitage des séries a pu se faire aussi bien directement à la suite de la première séric récurrente qu'à un stade ultérieur du débitage (tout comme il a pu ne pas s'effectuer).

L'exploitation du nuclćus s'est poursuivie dans quelques cas sur l'autre face, selon la même méthode ou par le débitage d'enlèvements non prédéterminés.

La méthode de débitage de l'abri Suard se caractérise donc par la production d'enlèvements unipolaires, production découpée séquentiellement en séries qui sont soit de même sens, soit opposées ou orthogonales entre elles (fig.2).

Quel que soit le schéma d'obtention, les éclats produits ne s'avèrent pas vraiment différents d'un point de vue morphologique : ils sont dans l'ensemble plutôt allongés (mais rarement laminaires), soit de morphologie quadrangulaire, soit moins fréquemment triangulaires ou à extrémité pointue. Selon leur place dans la série récurrente ils sont de section symétrique ou asymétrique. Les éclats Levallois présentent donc des caractères morphotechniques assez variés ; ils sont été largement retouchés puique près de $50 \%$ d'entre eux sont les supports d'outils variés. Il faut souligner que la retouche affecte autant les produits issus des autres phases de débitage (produits corticaux, indifférenciés et prédéterminants) et qu'aucune gestion préférentielle de certains produits en types d'outils distincts ne se dégage. 


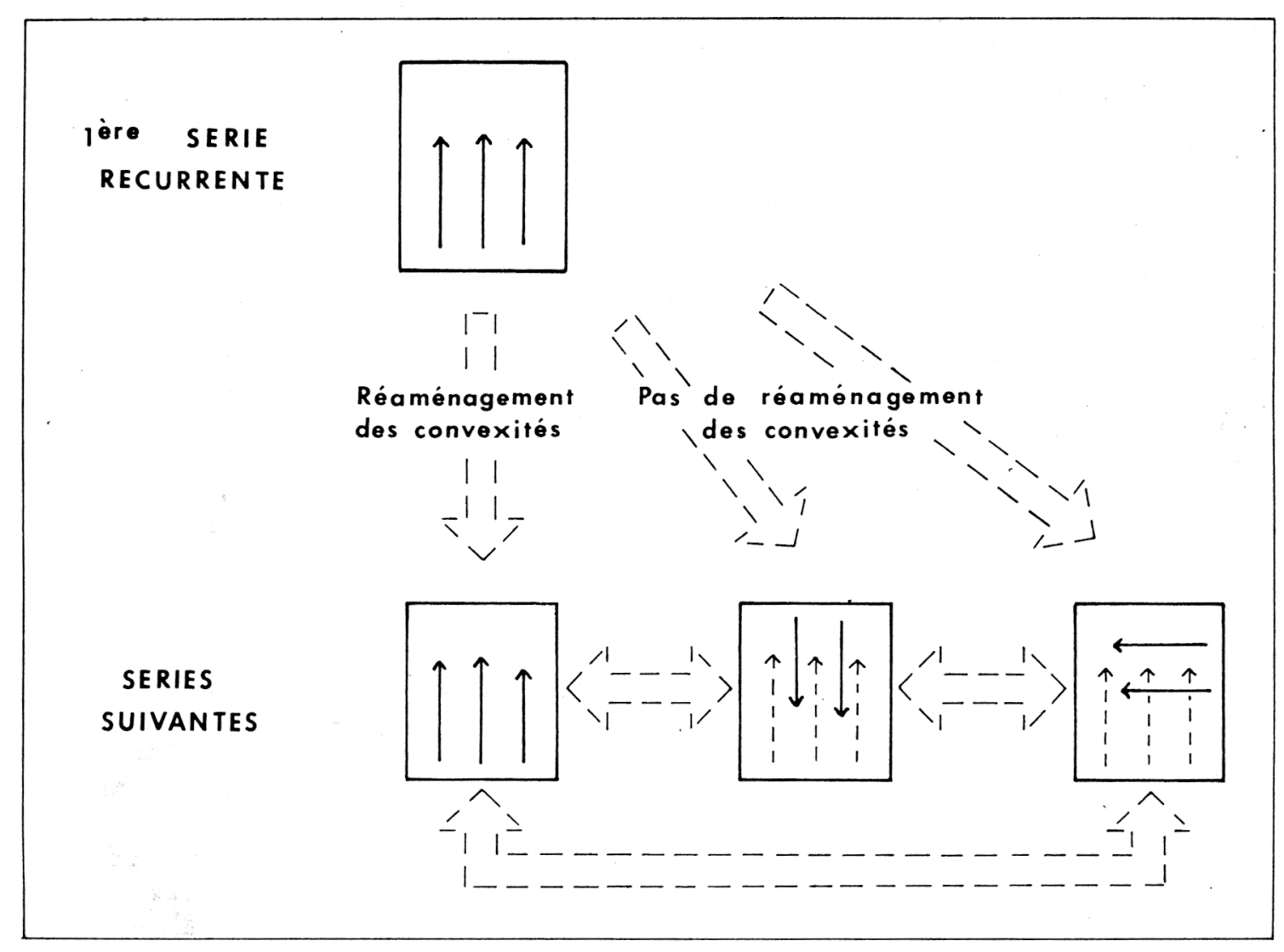

Fig. 2. Le principe de la méthode de débitage de l'abri Suard.

\section{INTERPRETATIONS ET COMPARAISONS.}

La méthode de débitage employée à l'abri Suard présente à la fois une grande rigidité dans son principe et une certaine souplesse dans sa réalisation.

La rigidité réside dans la notion d'unipolarité qui conditionne l'ensemble du débitage ; cette notion paraît si fortement imprégnée dans l'esprit des tailleurs qu'elle caractérise non seulement les phases de plein débitage mais aussi les phases de mise en forme des nucléus.

Le débitage est strictement unipolaire au sein d'une même série récurrente, et de ce fait on peut sans difficulté rattacher cette méthode à la méthode Levallois récurrente unipolaire (Boëda, 1986a).

Par contre la souplesse intervient lorsque l'on considère non plus une seule série d'enlèvements, mais les différentes séries unipolaires dans leur enchaînement.

A l'issue d'une première série unipolaire, des changements d'orientation peuvent intervenir dans le débitage des séries ; ces changements semblent être motivés par un comportement technique assez opportuniste. En effet, si au sein d'un schéma unipolaire strict un réaménagement (même minime) des convexités sur la surface de débitage s'avère indispensable pour la réalisation de séries successives d'enlèvements, cela n'est pas le cas lorsque l'orientation du débitage change d'une série à l'autre. Dans ce cas le tailleur a semble-t-il profité de la configuration de la surface de débitage et des convexités restantes à la suite d'une série récurrente pour produire directement une deuxième série (voire même ensuite une troisième), orthogonale ou opposée à la précédente. Une grande souplesse régit l'enchaînement des séries : c'est selon l'évolution du débitage et dans le souci du maintien d'un certain équilibre de la surface de débitage que le tailleur a opté pour telle ou telle orientation tout en restant dans le champ restreint de séries d'enlèvements unipolaires.

Si le passage à des séries d'orientations différentes s'avère plus économique en termes d'entretien de la surface de débitage (par l'absence de remise en forme des convexités), il implique l'aménagement d'un nouveau plan de frappe à chaque changement de sens. Ainsi les opérations de mise en place des plans de frappe constituent des étapes fondamentales dans la séquence opératoire. En cela le plan de frappe apparaît comme un critère technique plus important dans le débitage de l'abri Suard que les convexités (conférant à la surface de débitage son aspect bombé) qui ont été plutôt négligées lors de la mise en forme des nucléus tout comme au cours des phases de plein débitage. 

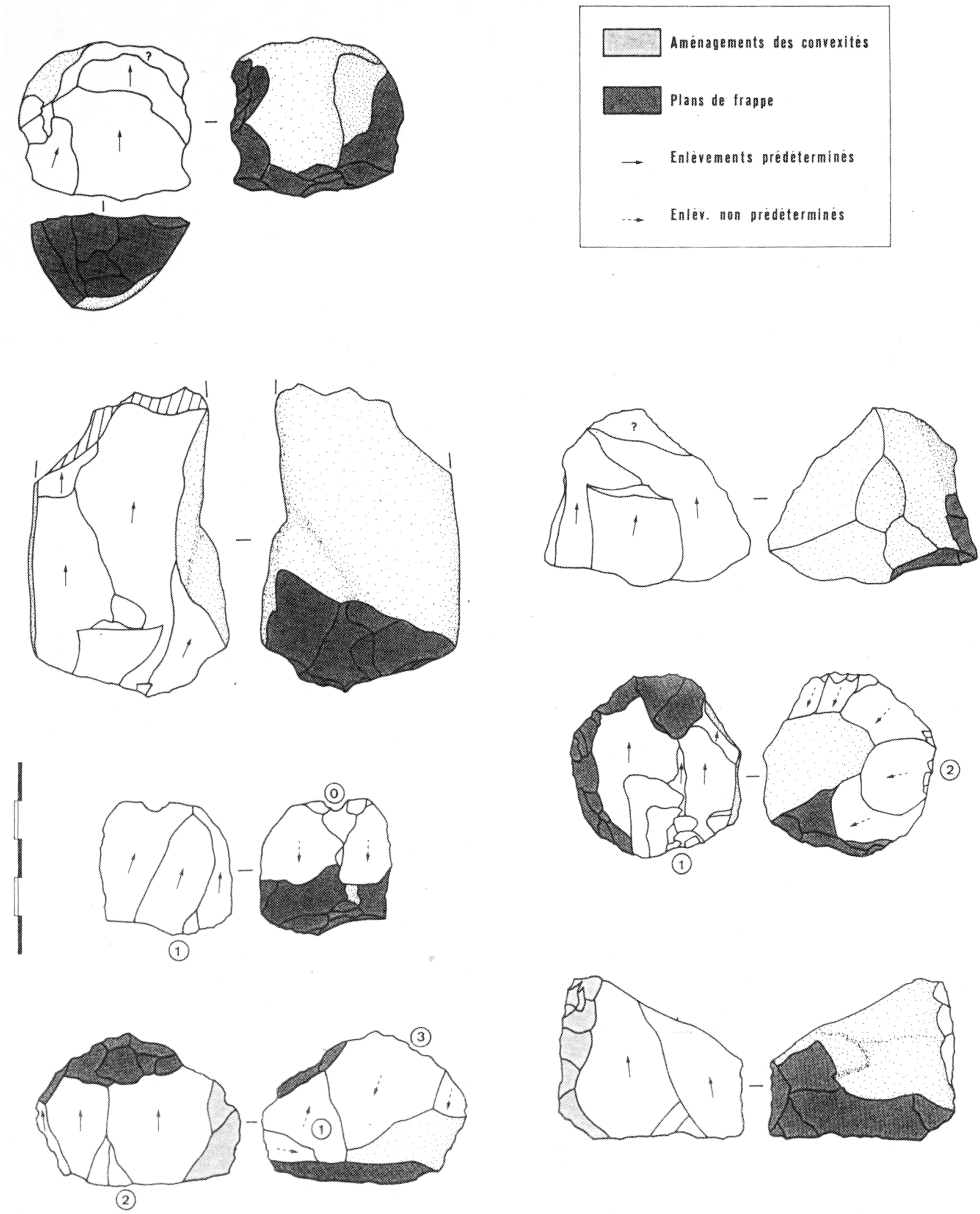

Fig. 3. Abri Suard, couche 51 : nucléus Levallois à séries récurrentes unipolaires. 

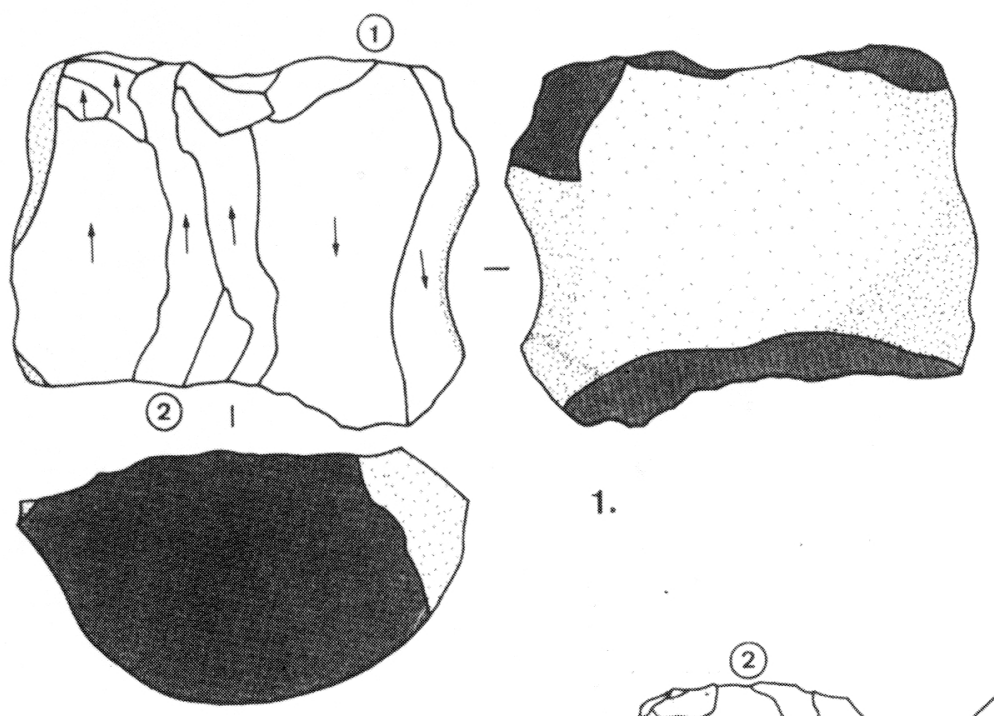

1.

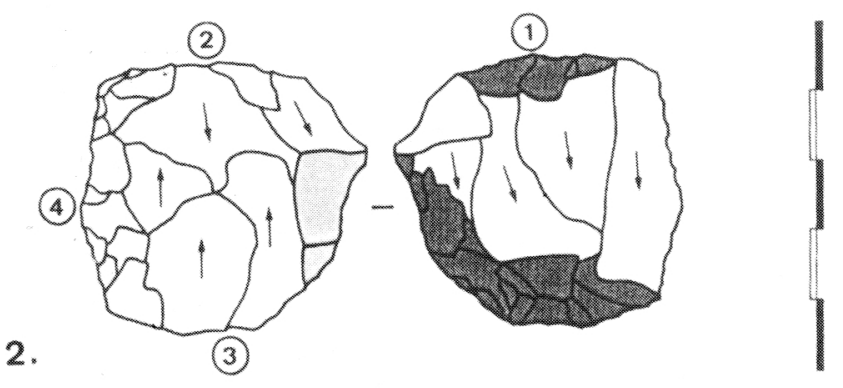

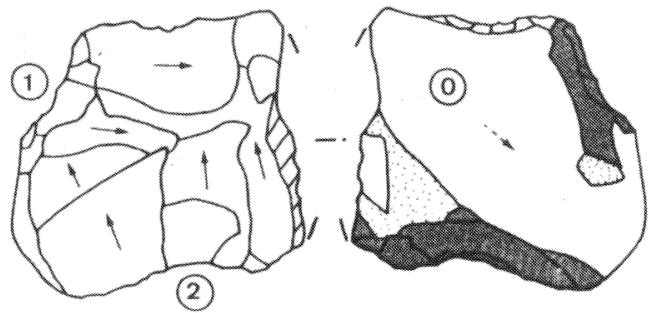

3.

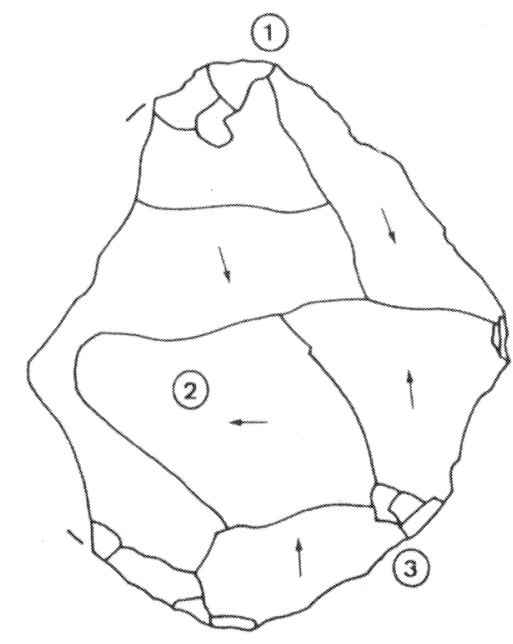

5.

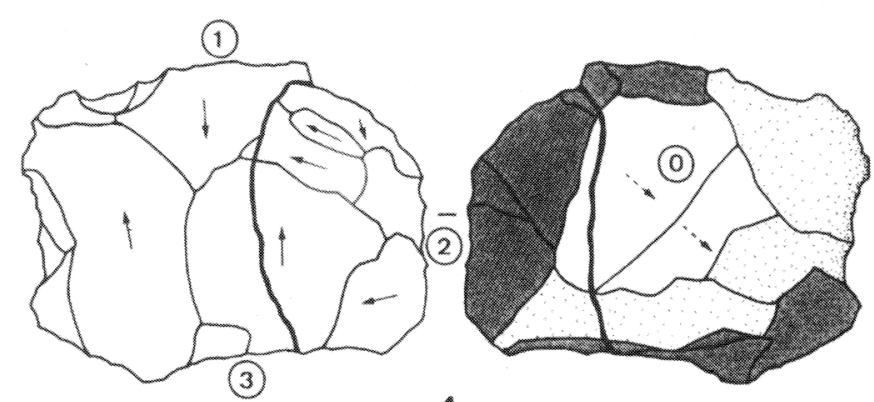

4.

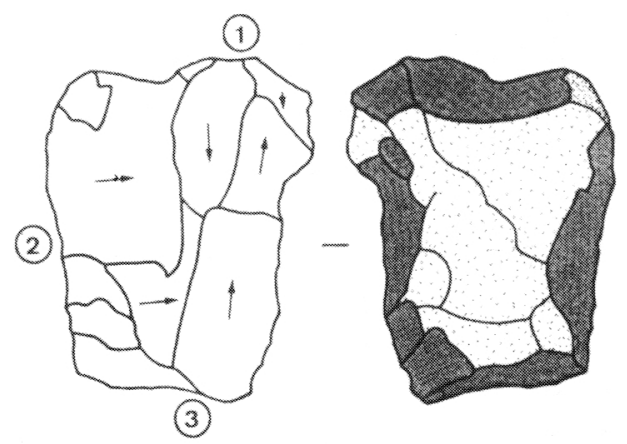

6.

Fig. 4. Abri Suard, couche 51 : nucléus Levallois à séries récurrentes bipolaires $\left(\mathrm{n}^{\circ} 1\right.$ et $\left.\mathrm{n}^{\circ} 2\right)$, et nucléus Levallois à séries récurrentes orthogonales ( 2 séries : $n^{\circ} 3,3$ séries : $n^{\circ} 4$ à $n^{\circ} 6$. 
Une telle méthode illustre clairement le fait que si les méthodes de débitage Levallois sont régies par des agencements assez rigides d'enlèvements, une certaine souplesse peut intervenir dans le déroulement du débitage.

Il semble d'ores et déjà que cette méthode $d$. débitage ne soit pas propre à l'abri Suard : une méthode semblable a été décrite pour le matériel du site moustérien des Ramandils (Aude) dont le remplissage est corrélé au Würm ancien (Sellet, 1989).

Elle se distingue par contre des méthodes unipolaires et bipolaires déctrites par Eric Boëda sur la base du matériel du niveau IIA de Biache-Saint-Vaast, correspondant chronologiquement au stade isotopique 7 (Sommé Tuffreau, Aitken et al., 1988) ; aux nucléus Levallois récurrents unipolaires et bipolaires correspondent dans ce site deux méthodes de débitage distinctes (Boëda, 1986 b et 1988 a).

Les couches VII et VIII de l'abri Vaufrey, corrélées au stade isotopique 6 (Delpech et Laville, 1988) se caractérisent par des méthodes similaires à celles de Biache avec une prédominance pour l'exploitation strictement unipolaire des nucléus (Geneste, 1985 et 1988).

A l'abri Suard l'exploitation des nucléus se fait par séries successives qui peuvent être de même orientation ou d'orientations différentes; il en résulte des nucléus unipolaires (fig. 3), bipolaires et orthogonaux (fig. 4), découlant des mêmes processus de mise en forme et s'intégrant dans la même séquence opératoire.

Ainsi la reconstitution de la séquence de débitage, débouchant sur une vision dynamique de l'ensemble des opérations techniques peut permettre de regrouper au sein d'une même méthode des nucléus variés.

Corrélativement les méthodes de débitage peuvent donc présenter une certaine variabilité : à partir d'un principe de base qui reste le même, des adaptations ou options diverses sont susceptibles d'intervenir. Dans le cas de l'abri Suard la variabilité à l'intérieur de la méthode récurrente unipolaire découle tout autant d'options intervenant pendant les phases de plein débitage que d'options liées aux phases de mise en forme et d'entretien des nucléus:

- les variantes observées dans la direction de débitage dénotent une certaine souplesse intervenant dans l'enchaînement des séries au cours de la production ; - cette souplesse est liée par ailleurs à l'économie de l'entretien des convexités sur la surface de débitage. Les convexités font l'objet d'un investissement technique réduit en règle générale à l'abri Suard (que ce soit pour leur mise en place initiale ou pour leur réaménagement). Grâce à la sélection de formes propices et à l'utilisation d'un schéma opératoire opportuniste la conception et l'exploitation volumétrique caractéristiques du concept Levallois sont néanmoins présentes.

Les deux critères de prédétermination que sont les plans de frappe et les convexités sur les surfaces de débitage ont donc vraisemblablement des importances inégales selon les assemblages. Ils interviennent de ce fait activement dans la variabilité des méthodes de débitage Levallois.

I. cur rôle est primordial car ils influent directement (i...t autant que l'agencement des enlèvements prédéterminis) sur les caractères de la production. Une place conséquente mérite donc de leur être consacrée dans les analyses technologiques.

La reconstitution des enchaînements techniques dans la séquence de débitage de l'abri Suard a permis de mettre en évidence une méthode de débitage Levallois assez originale, présentant une certaine souplesse dans sa réalisation ; elle contribue à renforcer la notion de variabilité à l'intérieur du concept Levallois :

- la variabilité se manifeste non seulement par la présence de méthodes de débitage diffférenciées mais peut aussi entrer en jeu au sein d'une même méthode ;

- une variabilité se dégage aussi dans les modalités de mise en forme et d'entretien des nucléus.

Par ailleurs le matériel de l'abri Suard relève d'un débitage complexe et structuré. Cette constatation corrobore les données actuellement disponibles sur les phases anciennes du Paléolithique moyen, qu'elles soient fondées sur des analyses typologiques ou sur des approches technologiques.

Si l'on se réfère à ce dernier type de données, peut-on appréhender des relations entre les différentes méthodes de débitage Levallois et le contexte chronologique du Paléolithique moyen en Europe occidentale ? Autrement dit existe-t-il des stades évolutifs du débitage Levallois en rapport avec des méthodes de production distinctes?

L'idée déjà avancée (Geneste, 1990) d'une antériorité des méthodes unipolaires et bipolaires par rapport aux autres méthodes Levallois semble renforcée par les données de l'analyse technologique du matériel de l'abri Suard ; ces méthodes caractérisent la plupart des assemblages lithiques moustériens de l'Avant-Dernier Glaciaire en Europe occidentale et notamment Biache-Saint-Vaast et la grotte Vaufrey. Néanmoins il paraît prématuré d'essayer de dégager de véritables tendances, étant donné que les analyses technologiques, malgré leur développement actuel, concernent encore un nombre réduit de gisements. 


\section{BIBLIOGRAPHIE}

BLACWELL, B. SCHWARCZ, H.P. et DEBENATH, A. 1983. Absolute dating of hominids and palaeolithic artifacts of the cave of La-Chaise-de-Vouthon (Charente), France. Journal of Archaeological Science, 10, p. 493513.

BOEDA,E. 1986a. Approche technologique du concept Levallois et évaluation de son champd' application : étude de trois gisements saaliens et weichséliens de le France. septentrionale. 2 vol. : 385 p. 49 pl. Thèse : Université de Paris $\mathrm{X}$.

BOEDA, E. 1986b. Le débitage Levallois de BiacheSaint-Vaast (Pas-de-Calais) : première étude technologique. In : Chronostratigraphie et faciès culturels du Paléolithique inférieur et moyen dans l'Europe du NordOuest, actes du coll. international, Lillemons, sept. 1984 / organ. par A. Tuffreau et J. Sommé. Paris : S.P.F. : A.F.E.Q., 1986. p. 209-218 (Bull. de l'A.F.E.Q. Supplément ; 26).

BOEDA, E. 1988a. Analyse technologique du débitage du niveau II a. In : Le gisement paléolithique moyen de Biache-Saint-Vaast (Pas de Calais), vol. 1 : stratigraphie, environnement, études archéologiques (1ère partie) sous la dir. de A. Tuffreau et J. Sommé. Paris : S.P.F., 1988. p. 185-214 (Société Préhistorique Française. Mémoires ; 21).

BOEDA, E. 1988b. Le concept Levallois et évaluation de son champ d'application. In : L'Homme de Néanderthal, 4 : La technique, actes du coll. international de Liège, déc. 1986 / éd. par M. Otte. Liège : ERAUL, 1988. p. 13-26 (ERAUL ; 31).

BORDES, F. 1980. Le débitage Levallois et ses variantes. Bull. S.P.F., 77, 2, p. 45-49.

DAVID, P.et PRAT, F. 1965. Considérations sur les faunes de La Chaise (commune de Vouthon, Charente), abris Suard et Bourgeois-Delaunay. Bull. A.F.E.Q. , p. 222-231.

DEBENATH, A. 1965. Recherches sédimentologiques sur le remplissage des grottes-abris de La - Chaise -de Vouthon (Charente). 168 p. Thèse de 3ème cycle: Géologie : Bordeaux.

DEBENATH, A. 1974. Recherches sur les terrains quaternaires charentais et les industries qui leurs sont associées. 2 vol. : 678 p. , 209 pl. Thèse de Doctorat d'Etat: Sciences Naturelles : Université de Bordeaux I.
DEBENATH, A. et DELAGNES, A. A parâttre. L'abri Suard (La Chaise-de-Vouthon, Charente) dans le contexte des industries du Pléistocène moyen : une mise au point d'après les données de l'outillage. In : Les moustériens Charentiens : coll. international, Brive-la-Gaillarde, 2629 août 1990.

GENESTE, J.M. 1985. Analyse lithique d'industries moustériennes du Périgord : une approche technologique du comportement des groupes humains au Paléolithique moyen. 2 vol. : 567 p., 115 pl. Thèse : Université de Bordeaux I.

GENESTE, J.M. 1988. Les industries de la grotte Vaufrey : technologic du débitage, économie et circulation de la matière première lithique. In : La grotte Vaufrey, paléoenvironnement, chronologie, activités humaines / sous la dir. de J.P. Rigaud. Paris : S.P.F., 1988. p. 441-517 (Société Préhistorique Française. Mémoires ; 19).

GENESTE, J.M. 1990. Développement des systèmes de production lithique au cours du Paléolithique moyen en Aquitaine septentrionale. In : Paléolithique moyen récent et $P$ aléolithique supérieur ancien en Europe, actes du colloque international de Neumours, mai 1988/sous la dir. de C. Farizy. Nemours : A.P.R.A.I.F., 1990. p. 203-213 (Musée de Préhistoire d'Ile-de-France. Mémoires ; 3).

SCHWARCZ, H.P. et DEBENATH, A. 1979. Datation absolue des restes humains de la Chaise-de-Vouthon (Charente) au moyen du déséquilibre des séries d'uranium. C.R. Acad. Sc. Paris, 288, p.1155-1157.

SELLET, F. 1989. Gisement moustérien des Ramandils (Port-la-Nouvelle, Aude) : approche technologique des méthodes de débitage. 66p. Mémoire de D.E.A. : «Quaternaire : Géologic, Paléontologie Humaine, Préhistoire» : Muséum National d'Histoire Naturelle.

SOMME, J. TUFFREAU, A. AITKEN, M.J. et al. 1988. Chronostratigtraphie, climats et environnement. In : Le gisement paléolithique moyen de Biache-Saint-Vaast (Pasde-Calais), vol. 1 : stratigraphie, environnement, études archéologiques (1ère partie) / sous la dir. de A. Tuffreau et J. Sommé. Paris : S.P.F., 1988. p. 115-119 (Société Préhistorique Française. Mémoires ; 21). 